\title{
Simultaneous \\ Incidence of \\ Diaphragmatic Hernia and Intestinal \\ Perforation in a Cat
}

Seyedsadra Izadi ${ }^{1}$, Maryam Zabihi ${ }^{2}$,

Mohammad Sadegh Ghodrati ${ }^{3}$,

Mohammad Amin Reisdanaei ${ }^{1}$,

Seyed Reza Ghiasi ${ }^{4}$

THIS ABSTRACT HAS BEEN WITHDRAWN

BY THE AUTHOR

\section{Accidental fentanyl exposure in pets}

\section{Fiona Finlay ${ }^{1}$, James Dunne ${ }^{2}$ \\ 1 Virgin care, Bath, United Kingdom \\ 2 Wiltshire CCG, Devizes, United Kingdom}

\section{OBJECTIVES}

Fentanyl is a highly potent opioid - accidental transdermal exposure resulting in fatalities has been reported in pets and children. Discussion of the topic prompted us to review the literature

\section{METHODS}

Literature review

\section{RESULTS}

- Many human patients are prescribed fentanyl - a 25 microgram/hour fentanyl patch is equivalent to an oral morphine dose of 60-90mg in 24 hours

- Discarded patches may still contain $50 \%$ of original drug - enough to cause serious harm/death
- Used patches should be disposed of carefully and not be put in a wastepaper bin where pets may find and ingest them - there is more than a 30-fold increase in absorption from the buccal mucosa if chewed compared with the transdermal route

- Swallowing an intact patch results in less rapid drug release compared with chewing, but systemic absorption is still significant

- Patches may also accidentally stick to an animal's skin

- Discarded patches should be folded putting the sticky sides of each patch together so that it sticks to itself. They should then be wrapped in paper or plastic before disposal

- Patients should check regularly that patch has not fallen off. May cover it with adhesive film to ensure it stays on

- Identifying signs of fentanyl exposure in animals is challenging as many are non-specific eg excitement, pacing, panting

- Police sniffer dogs are at risk of fentanyl exposure and in some forces dog handlers are trained in the administration of naloxone

\section{STATEMENT (CONCLUSIONS)}

Veterinary surgeons should ask about fentanyl exposure in pets were there are concerns re symptoms. 